\title{
Pericardial Effusion with Tamponade Physiology in a Patient with Multiple Myeloma
}

\author{
Sairamya Bodempudi, MD \\ Thomas Jefferson University, sairamya.bodempudi@jefferson.edu \\ Rukaiya Bashir-Hamidu, MD \\ Thomas Jefferson Univeristy, rukaiya.bashir-hamidu@jefferson.edu \\ Preya Simlote, MD \\ Thomas Jefferson University, preya.simlote@jefferson.edu \\ Shuwen Lin, MD \\ Thomas Jefferson University, shuwen.lin@jefferson.edu \\ Philip Margiotta, MD \\ Thomas Jefferson University, philip.margiotta@jefferson.edu \\ Follow this and additional works at: https://jdc.jefferson.edu/tmf \\ Part of the Internal Medicine Commons

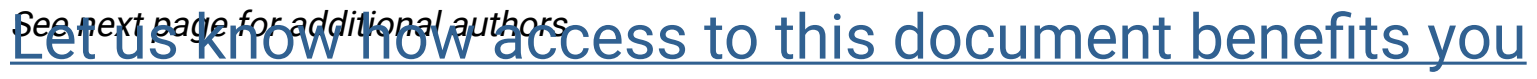

\section{Recommended Citation}

Bodempudi, MD, Sairamya; Bashir-Hamidu, MD, Rukaiya; Simlote, MD, Preya; Lin, MD, Shuwen; Margiotta, MD, Philip; Boortalary, MD, Tina; and Marhefka, MD, Gregary D. (2020) "Pericardial Effusion with Tamponade Physiology in a Patient with Multiple Myeloma," The Medicine Forum: Vol. 21 , Article 4. DOI: https://doi.org/10.29046/TMF.021.1.006

Available at: https://jdc.jefferson.edu/tmf/vol21/iss1/4

This Article is brought to you for free and open access by the Jefferson Digital Commons. The Jefferson Digital Commons is a service of Thomas Jefferson University's Center for Teaching and Learning (CTL). The Commons is a showcase for Jefferson books and journals, peer-reviewed scholarly publications, unique historical collections from the University archives, and teaching tools. The Jefferson Digital Commons allows researchers and interested readers anywhere in the world to learn about and keep up to date with Jefferson scholarship. This article has been accepted for inclusion in The Medicine Forum by an authorized administrator of the Jefferson Digital Commons. For more information, please contact: JeffersonDigitalCommons@jefferson.edu. 


\section{Pericardial Effusion with Tamponade Physiology in a Patient with Multiple Myeloma}

\section{Authors}

Sairamya Bodempudi, MD; Rukaiya Bashir-Hamidu, MD; Preya Simlote, MD; Shuwen Lin, MD; Philip Margiotta, MD; Tina Boortalary, MD; and Gregary D. Marhefka, MD 


\section{Pericardial Effusion with Tamponade Physiology in a Patient with Multiple Myeloma}

Sairamya Bodempudi MD, Rukaiya Bashir Hamidu MD, Preya Simlote MD, Shuwen Lin MD, Philip Margiotta MD, Tina Boortalary MD, Gregary D. Marhefka MD
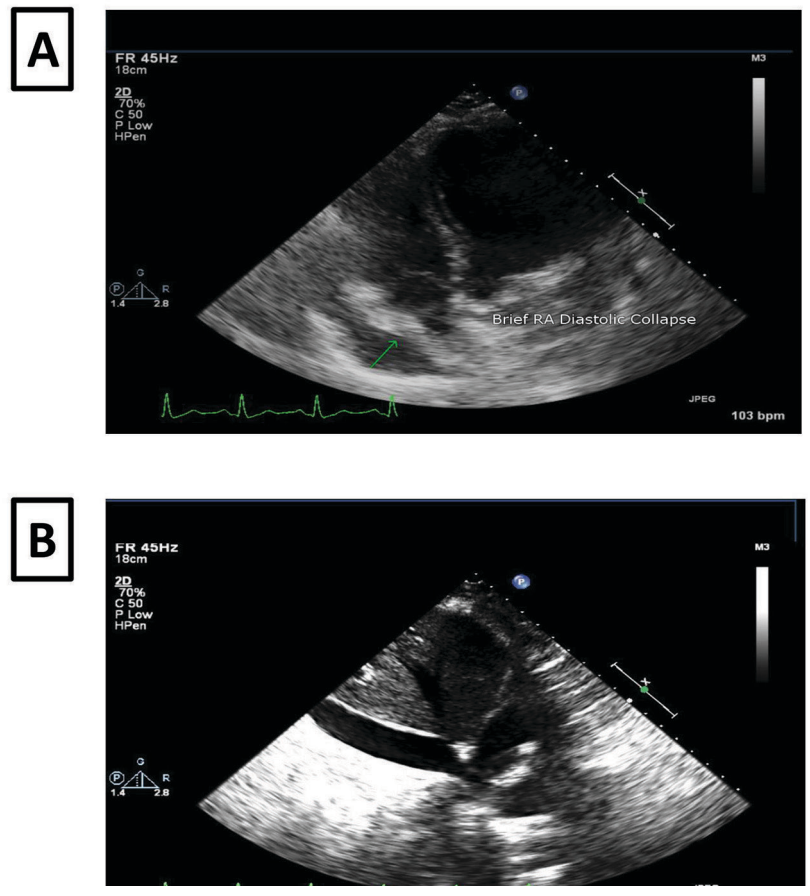

\section{INTRODUCTION}

Both solid and hematological malignancies are known to cause pericardial effusions. The hematological malignancies that most often cause pericardial effusion are leukemias, Non-Hodgkin's lymphomas, and Hodgkin's lymphomas. ${ }^{1}$ Patients can also develop pericardial effusions from cancer-related causes, such as chemotherapy, immunotherapy, radiation, or infection. ${ }^{2}$ Although it has been reported in the literature (approximately 27 cases between 1970 to 2019), malignant pericardial effusion from multiple myeloma is relatively uncommon. ${ }^{3.4}$ In addition, a rare but known complication of poorly controlled multiple myeloma is hyperviscosity syndrome, which typically presents as spontaneous hemorrhage of the mucosal membranes, headaches or neurological symptoms. We present a case of a patient with multiple myeloma who was found to have a pericardial effusion with tamponade that was also complicated by hyperviscosity syndrome.

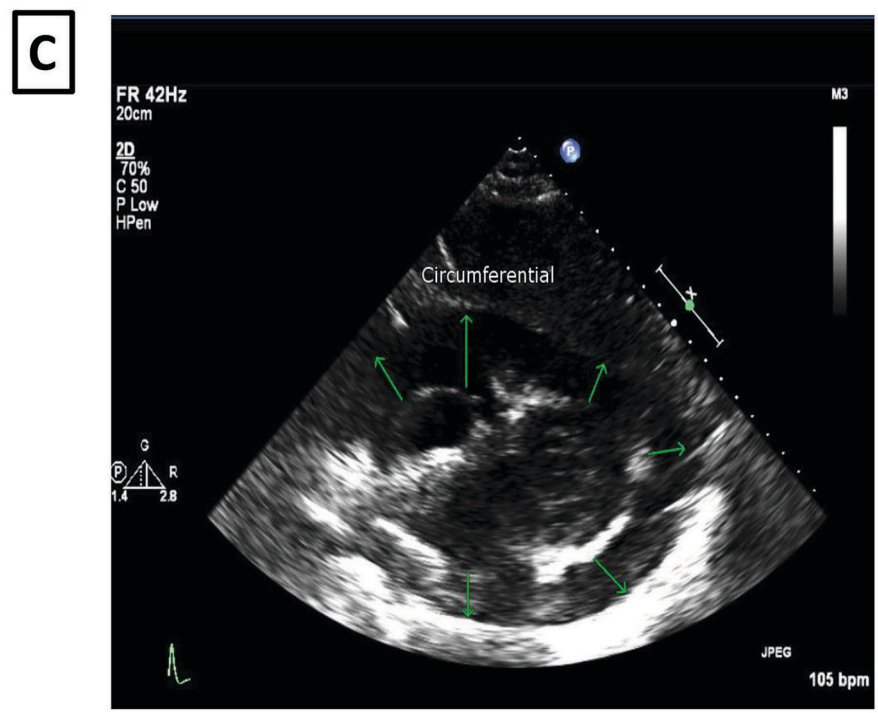

Figure 1. (A) RA diastolic collapse showing evidence of early tamponade. (B) Plethoric IVC from a subcostal view. (C) Circumferential shot from a subcostal view to show the extent of the pericardial effusion.

\section{CASE PRESENTATION}

\section{History of Present Illness}

A 78-year old African American female with a past medical history of IgA Kappa Multiple Myeloma was transferred to the Cardiovascular Intensive Care Unit (CVICU) at Thomas Jefferson University Hospital (TJUH) after being diagnosed with a pericardial effusion with tamponade physiology at an outside hospital.

The patient was diagnosed with multiple myeloma 3 years prior on a left maxillary biopsy revealing a plasmacytoma. Due to medical complications, multiple hospitalizations, poor follow up, and intermittent refusal of chemotherapy, the patient never achieved remission of her malignancy. Due to continued progression of her disease, she was started on Melphalan/methylprednisolone therapy and had finished 5 cycles. 
The patient was in her usual state of health until one week prior to presentation when she developed a cough and dyspnea on exertion. Since her symptoms progressed, she eventually went to the emergency department, where she was noted to have a pericardial effusion with signs of early tamponade physiology. She was transferred to the CVICU at TJUH for further care.

Upon arrival to $\mathrm{TJUH}$, the patient was tachycardic with heart rates in the 110 s. A physical exam revealed jugular venous distension, normal S1 and S2 heart sounds, slight end expiratory wheezing throughout the lung fields, and a droopy right eyelid with nodules. A pulsus paradoxus was found to be $5 \mathrm{~mm} \mathrm{Hg}$ and a bedside transthoracic echocardiogram (TTE) showed a circumferential pericardial effusion with a maximum size of $2.2 \mathrm{~cm}$, brief right atrial diastolic collapse, and a non-compressible inferior vena cava concerning for early tamponade physiology.

\section{Hospital Course}

Due to concern for tamponade, the patient received a pericardiocentesis with pericardial drain placement. Approximately $185 \mathrm{~mL}$ of sanguineous fluid was drained and sent to the lab for typical studies, including cytology plus flow cytometry. A repeat TTE showed trivial residual pericardial effusion with improved hemodynamic profile and resolution of tamponade. Pericardial fluid cytology and flow cytometry results showed evidence of plasma cells consistent with her underlying plasma cell myeloma.

Within a day of admission, the patient developed significant interval swelling of her right eye with ecchymosis of her medial upper eyelid and significant conjunctival hemorrhage. Due to subjective shortness of breath, a chest radiograph was done and showed the correct positioning of the pericardial drain. However, it incidentally showed new left sided pleural effusion. Given multiple sites of spontaneous hemorrhage and significantly elevated IgA levels (>4500), there was concern for hyperviscosity syndrome. Medical oncology was consulted and recommended urgent plasmapheresis, however, the patient initially refused conveying her wishes to pursue non-aggressive medical therapy. She was temporized with intravenous fluids and high-dose steroids.

On day 7 of post-pericardial drain placement, there continued to be high output ( $>75 \mathrm{~mL} /$ day) from the drain. Given the increased risk of infection with prolonged drain placement and continued high output in the setting of hyperviscosity syndrome, the decision was made for doxycycline sclerotherapy to the pericardium with subsequent removal of the pericardial drain. Repeat TTE the following day showed only trivial pericardial effusion.
Eventually, the patient consented to plasmapheresis, resulting in a downtrend of her IgA level. She received cytoreductive chemotherapy with cyclophosphamide and dexamethasone. The patient's post-chemotherapy hospital course was complicated by paroxysmal supraventricular tachycardia, acute kidney injury, stressinduced hyperglycemia requiring insulin therapy and febrile neutropenia from an unknown infectious source. These complications resolved with medical management.

Prior to discharge, goals of care were readdressed. Although initially hesitant, the patient and her family ultimately decided to pursue aggressive treatment of her multiple myeloma. She was subsequently discharged to a sub-acute rehabilitation center after which she underwent chemotherapy with daratumumab, pomalidomide, and dexamethasone. Two months after the patient's initial presentation to the TJUH CVICU, the patient is doing well on her current chemotherapy regimen with down trending IgA levels. She has stable pleural effusions on chest x-ray. Repeat TTE's show no evidence of pericardial effusion. She remains symptom free with no further medical complications.

\section{DISCUSSION \& CONCLUSIONS}

As stated previously, pericardial effusions secondary to multiple myeloma are very rare, seen in $<1 \%$ of cases. ${ }^{4}$ Although in our case, the pericardial fluid was determined to be malignant in origin based on flow cytometry and cytology, the rapid re-accumulation of pericardial effusion almost a week after drain placement was presumed to be due to the spontaneous bleeding complications of hyperviscosity syndrome. The medical interventions were unique for this particular patient due to her preference of treatments. She was initially temporized with fluids and dexamethasone instead of immediately being started on plasmapheresis. Interestingly, temporizing measures did initially slow the rate of drain output.

Despite stabilizing with steroids and fluids, the patient's re-accumulating pericardial effusion necessitated management with sclerotherapy. Per review of literature, doxycycline sclerotherapy for recurrent pericardial effusions secondary to multiple myeloma has not been performed. ${ }^{4}$ The decision to attempt bedside doxycycline sclerotherapy highlights the role of shared-decision making between the patient, her family, cardiology, medical oncology, and thoracic surgery in order to respect the patient's wishes to opt for minimally invasive treatment strategies. 


\section{Bodempudi, MD et al.: Pericardial Effusion}

Our case also explores the complicated relationship between management of plasma cell pericardial effusions and hyperviscosity syndrome, which may increase the risk of bleeding into the pericardium. When the patient is clinically and hemodynamically stable, and willing to receive plasmaphoresis, it is reasonable to treat the hyperviscosity syndrome prior to draining the pericardium to decrease the risk of post-intervention bleeding. However, when a patient presents with early signs of tamponade physiology or hemodynamic instability, then a pericardiocentesis could be considered and prioritized with simultaneous temporization of hyperviscosity syndrome utilizing high-dose steroids and fluids as we had done in this case. Finally, the case demonstrates that it is essential to intermittently revisit goals of care because our patient's wishes evolved and molded her medical management. On-going discussions resulted in her changing her initial decision, and altering the course of her disease process. Our case of a patient with multiple myeloma presenting with malignant pericardial effusion as an extra-medullary manifestation of chemotherapy-refractory multiple myeloma is therefore unique from a clinical presentation, medical intervention, and a shared decision-making standpoint.

\section{REFERENCES}

1. Burazor, I., Imazio, M., Markel, G., \& Adler, Y. (2013). Malignant Pericardial Effusion. Cardiology, 124(4), 224-232. doi: 10.1159/000348559

2. Szturmowicz, M., Pawlak-Cieślik, A., Fijałkowska, A., Gątarek, J., Skoczylas, A., Dybowska, M.Tomkowski, W. (2017). The value of the new scoring system for predicting neoplastic pericarditis in the patients with large pericardial effusion. Supportive Care in Cancer, 25(8), 2399-2403. doi: 10.1007/s00520017-3645-4

3. Pan, J., Chen, J., Filicko, J., Grosso, D., Nagurney, M., Alpdogan, O., \& Flomenberg, N. (2017). Relapsed Multiple Myeloma Presenting as Intracranial Plasmacytoma and Malignant Pericardial Effusion following Recent Allogeneic Stem Cell Transplantation. Case Reports in Oncology, 10(2). 582-587. doi: 10.1159/000478001

4. Skipina TM, Sane DC, Cui C, Song S, Phillips SG, Jarrett RW. (2019). A plasma cell-based pericardial effusion leading to tamponade in a patient with multiple myeloma - a case report and review of the literature. Cardiovascular Pathology, 40:41-46. doi:10.1016/j.carpath.2019.02.002. 